factor-23. Nephrol Dial Transplant. 2008;23:827-833. 21. Ichikawa $S$, et al. A homozygous missense mutation in human KLOTHO causes severe tumoral calcinosis. J Clin Invest. 2007;117(9):2684-2691.
22. Farrow EG, et al. Iron deficiency drives an autosomal dominant hypophosphatemic rickets (ADHR) phenotype in fibroblast growth factor-23 (Fgf23) knock-in mice. Proc Natl Acad Sci U S A. 2011;
108(46):E1146-E1155.

23. Farrow EG, Davis SI, Summers LJ, White KE. Initial FGF23-mediated signaling occurs in the distal convoluted tubule. J Am Soc Nephrol. 2009;20(5):955-960.

\title{
Understanding the TXA seizure connection
}

\author{
Debra A. Schwinn, ${ }^{1}$ G. Burkhard Mackensen, ${ }^{2}$ and Emery N. Brown ${ }^{3}$
}

\begin{abstract}
1Department of Anesthesiology, Pharmacology, and Biochemistry, Roy J. and Lucille A. Carver College of Medicine, The University of lowa, lowa City, lowa, USA. 2Department of Anesthesiology and Pain Medicine, University of Washington, Seattle, Washington, USA. ${ }^{3}$ Department of Brain and Cognitive Sciences, MIT-Harvard Division of Health Science and Technology, Massachusetts Institute of Technology, Cambridge, Massachusetts, USA.
\end{abstract}

\begin{abstract}
Transexamic acid (TXA) is an antifibrinolytic that has been used successfully to prevent blood loss during major surgery. However, as its usage has increased, there have been growing reports of postsurgical seizure events in cardiac surgery patients. In this issue of the JCI, Lecker et al. explore this connection and suggest that TXA-mediated inhibition of glycine receptors may underlie the effect. This finding prompted the authors to explore the preclinical efficacy of common anesthetics that function by reducing the TXA-mediated inhibition to prevent or modify postsurgical seizures.
\end{abstract}

\section{Introduction}

Translation from clinical bedside to laboratory bench and back is the holy grail of medicine. In this issue, Lecker et al. (1) undertake just such a translation. In an elegant set of studies, the authors investigate the neuronal mechanisms that underlie seizures occurring as side effects in patients receiving transexamic acid (TXA) to reduce blood loss during major cardiovascular surgery. Given the structural similarity between the antifibrinolytic drug TXA and inhibitory neurotransmitter-gated $\mathrm{Cl}^{-}$channel glycine receptors, the authors tested the hypothesis that TXA inhibits glycine receptor action as a possible mechanism underlying the seizures. After demonstrating that TXA inhibits glycine receptors, they then proposed that anesthetic agents with glycine receptor agonist properties may be uniquely suited to prevent such seizures. The studies presented are of high quality, based on an impressive range of laboratory data and models. However, they may be premature in recommending specific clinical actions to prevent seizures based on the limited clinical data. To place these interesting and provocative translational findings in context, we review use of antifibrinolytics in major cardiovascular surgery; this is followed by a brief overview of mechanisms of general anesthesia rec-

Conflict of interest: Debra A. Schwinn is a consultant for Watson Pharmaceuticals.

Citation for this article: J Clin Invest. 2012; 122(12):4339-4341. doi:10.1172/JCI66724 ognized to date and how anesthetic agents may play a role in treating seizures generally rather than specifically. We conclude by suggesting how the work of Lecker et al. moves the field forward, and where further work is still needed.

\section{Antifibrinolytic agents and major cardiovascular surgery}

Excessive perioperative bleeding remains a complication of cardiac surgery employing cardiopulmonary bypass, with blood transfusions considered a major contributor to subsequent morbidity and mortality (2). Despite serious efforts to achieve perioperative blood conservation by multimodal approaches (e.g., point-of-care monitoring paired with institution-specific blood transfusion algorithms and applied pharmacologic strategies), cardiac surgery patients continue to receive the vast majority of all perioperative blood transfusions (3). Therefore, antifibrinolytic therapy, because of its proven hemostatic effects, has become routine in this setting. Historically, three main antifibrinolytics have been used in cardiac surgery: lysine analogs $\varepsilon$-aminocaproic acid (EACA) and TXA in patients at low risk for bleeding, and the serine protease inhibitor aprotinin in patients at high risk for perioperative bleeding (such as those undergoing reoperations). However, with the removal of aprotinin from clinical practice following its association with a higher incidence of death and renal dysfunction in the Blood Conservation Using Anti-Fibrinolytics in a Randomized Trial (BART) study, many institutions worldwide have updated their therapeutic protocols $(4,5)$. The safety concerns surrounding aprotinin use that were raised in the BART study are largely backed by a recent systematic review and network meta-analysis confirming increased risk of mortality and renal morbidity with aprotinin versus TXA and EACA (6). The search for alternatives to aprotinin recently culminated in a phase 2 clinical trial demonstrating the inferiority of the novel protease inhibitor ecallantide in combating blood loss during high-risk cardiac surgery compared with TXA, and enhanced hemostatic efficacy with higher doses of TXA (7). However, as TXA usage has increased, there has been a growing awareness that some patients experience TXA-induced seizures. Occurrence peaks during the early postoperative period $(8)$ in a dose-related manner $(9,10)$, and seizures are more common in patients with a history of renal dysfunction (11).

\section{General anesthesia and seizures}

General anesthesia is critical for performing most surgical and many non-surgical interventions (12). This state is induced by administering an intravenous hypnotic such as propofol and maintained by a combination of inhaled drugs, hypnotics, opioids, and muscle relaxants. Understanding the mechanism whereby inhaled anesthetics create this drug-induced reversible coma has been a major area of investigation since the first use of ether in the 1840s. Substantial evidence has established that the molecular targets of inhaled anesthetic drugs in the brain and central nervous system are $\mathrm{GABA}_{\mathrm{A}}$, NMDA and glycine receptors, two-pore potassium channels, and HCN channels (13). Although immobility is now believed to result from inhaled anesthetics acting mostly at these targets in the spinal cord (14), the mechanisms of unconsciousness, amnesia, and analgesia 
are less well defined. In contrast, for propofol, which is known to act by enhancing $\mathrm{GABA}_{\mathrm{A}}$ inhibition, systems neuroscience analyses now provide highly plausible neural circuit details of how its actions at $\mathrm{GABA}_{\mathrm{A}}$ receptors in the cortex, thalamus, and brainstem can lead to unconsciousness and sedation (15).

Seizures, typically characterized as hypersynchronous neuronal activity due to unbalanced, uncontrolled excitation, arise through multiple mechanisms (16), and establishing the precise cause is often a challenge. Conditions such as fevers, infection, and brain trauma that enhance neural activity can make seizures more likely. One possible mechanism is loss of control in key GABAergic inhibitory circuits (16). The findings in the current study suggest that inactivation of glycine-mediated circuits, which are also inhibitory networks, may be an alternative mechanism for seizure induction. Although propofol and inhaled anesthetics are not first-line therapies for seizures, they are the last-line therapy for status epilepticus, as they place the patient in a drug-induced coma (17). This coma is typically induced and maintained by sustained delivery of sufficient doses of the anesthetic until the seizure activity, monitored by EEG, converts to a desired level of burst suppression. The presumed mechanisms of action are enhanced GABAergic inhibition (18), as well as metabolically induced opening of ATP-dependent potassium channels (19). The seizure activity is arrested, but the mechanism of the therapy may not relate to the mechanism of the seizure. Therefore, it is highly plausible, as suggested by Lecker and colleagues, that propofol or isoflurane could be used to treat seizures associated with TXA, especially since their in vitro findings suggest that these drugs can directly block glycinemediated activation induced by TXA.

\section{Unanswered questions}

Since TXA binds competitively to GABA type A receptors, it is perhaps no surprise that an association between TXA and postoperative seizures is found (20). However, it is unclear why most episodes of seizures occur during the immediate postoperative hours, a time when levels of intraoperatively administered TXA in the serum and cerebrum should already be declining. One possibility is that TXA induces modifications (neuroplasticity) in these inhibitory circuits that outlast its pharmacological time course. In addition, TXA is known to cross the blood-brain barrier (BBB), especially when $\mathrm{BBB}$ integrity is challenged. Thus, the higher incidence of TXA-related seizures in patients undergoing openheart procedures may relate to disruption of BBB integrity by cerebral emboli, which are known to be much more prevalent during open-heart procedures versus coronary artery bypass grafting (21). This may also explain why Lecker et al. found higherthan-expected TXA concentrations in CSF from a patient undergoing major aortic surgery, where BBB disruption is also common. Lecker et al. also demonstrated that TXA binds to glycine receptors, at least in mice (1). Thus, competitive antagonism of both glycine and $\mathrm{GABA}_{\mathrm{A}}$ receptors may serve as one plausible explanation for the association between TXA and postoperative seizures (20).

The next fundamental question is whether TXA-induced convulsions are true seizures or, as some researchers and caregivers report, dystonic movements more akin to those seen in strychnine poisoning. This latter possibility is intriguing given that strychnine is also a competitive glycine receptor antagonist (22). Unfortunately EMG and EEG recordings of the events are lacking due to unexpected timing of these incidents, and it is unknown whether patients have residual cognitive defects. Historically, patients who have seizures after cardiac surgery generally have higher incidence of postoperative neurologic complications, defined as stroke and delirium (8), but it is unclear whether these complications also follow TXA-induced seizures, which are generally easily treated.

Lecker et al. examined possible mechanisms to prevent TXA-induced seizures. Their study elegantly demonstrates that TXA inhibition of glycine receptors activated by a low concentration of glycine is reversed by isoflurane at clinically relevant concentrations, as well as to a lesser extent by propofol (1). Most patients receive sedation (such as continuous propofol infusion) until they are extubated postoperatively in the ICU, a process that may initially mask (or prevent) the occurrence of seizures.

\section{Summary}

In the article in this issue of the JCI, Lecker et al. provide new information on the mechanism underlying TXA-induced seizures, specifically elucidation of competitive inhibition by TXA of CNS-derived inhibitory glycine receptors. They also demonstrate that TXA inhibition of glycine receptors activated by a low concentration of glycine is reversed by isoflurane at clinically relevant concentrations, as well as to a lesser extent by propofol. These findings suggest that risk for TXA-induced seizures may be reduced by modifying (decreasing) TXA exposure by using a lower-dose regimen, or that seizures may be prevented with the use of isoflurane or propofol after surgery. Since early extubation and shorter ICU time after major surgery correlate with better patient outcome, it may not be practical to continue an anesthetized state for very long after surgery. However, anesthetics have multiple targets, and since most of their transmitted effects are inhibitory, it is likely that various combinations of drugs may work to provide the desired anticonvulsant effects. The use of agents that prevent glycine inhibition by TXA, as reported by the authors, certainly is one intriguing possibility.

\section{Acknowledgments}

The authors are funded by grants from NIH to D.A. Schwinn (HL49103) and E.N. Brown (DP1OD003646).

Address correspondence to: Debra A. Schwinn, Dean, Roy J. and Lucille A. Carver College of Medicine, Professor of Anesthesiology, Pharmacology and Biochemistry, The University of Iowa, 212 CMAB, 451 Newton Road, Iowa City, Iowa 52242-1101, USA. Phone: 319.384.4547; Fax: 319.353.5617; E-mail: debra-schwinn@uiowa.edu.

1. Lecker I, Wang D-S, Romaschin AD, Peterson M, Mazer CD, Orser BA. Tranexamic acid concentrations associated with human seizures inhibit glycine receptors. J Clin Invest. 2012;122(12):4654-4666.

2. Murphy GJ, Reeves BC, Rogers CA, Rizvi SI, Culliford L, Angelini GD. Increased mortality, postoperative morbidity, and cost after red blood cell transfusion in patients having cardiac surgery. Circulation. 2007;116(22):2544-2552.

3. Ferraris VA, et al. Perioperative blood transfusion and blood conservation in cardiac surgery: the Society of Thoracic Surgeons and The Society of Cardiovascular Anesthesiologists clinical practice guideline. Ann Thorac Surg. 2007;83(5 suppl):S27-S86.

4. Mangano DT, Tudor IC, Dietzel C. The risk associated with aprotinin in cardiac surgery. N Engl J Med. 2006;354(4):353-365.

5. Fergusson DA, et al. A comparison of aprotinin and lysine analogues in high-risk cardiac surgery. NEngl J Med. 2008;358(22):2319-2331.

6. Hutton B, Joseph L, Fergusson D, Mazer CD, Shapiro S, Tinmouth A. Risks of harms using antifibrinolytics in cardiac surgery: systematic review and network meta-analysis of randomised and observational studies. Brit Med J. 2012;345:e5798.

7. Bokesch PM, et al. A phase 2 prospective, randomized, double-blind trial comparing the effects of tranexamic acid with ecallantide on blood loss from high-risk cardiac surgery with cardiopulmonary bypass (CONSERV-2 Trial). J Thorac Cardiovasc Surg. 2012;143(5):1022-1029. 
8. Manji RA, Grocott HP, Leake J, et al. Seizures following cardiac surgery: the impact of tranexamic acid and other risk factors. Can J Anaesth. 2012; 59(1):6-13.

9. Murkin JM, Falter F, Granton J, Young B, Burt C, Chu M. High-dose tranexamic acid is associated with nonischemic clinical seizures in cardiac surgical patients. Anesth Analg. 2010;110(2):350-353.

10. Kalavrouziotis D, Voisine P, Mohammadi S, Dionne $\mathrm{S}$, Dagenais F. High-dose tranexamic acid is an independent predictor of early seizure after cardiopulmonary bypass. Ann Thorac Surg. 2012;93(1):148-154.

11. Montes FR, Pardo DF, Carreno M, Arciniegas C, Dennis RJ, Umana JP. Risk factors associated with postoperative seizures in patients undergoing cardiac surgery who received tranexamic acid: a casecontrol study. Ann Card Anaesth. 2012;15(1):6-12.

12. Brown EN, Lydic R, Schiff ND. General anesthesia, sleep, and coma. N Engl J Med. 2010;
363(27):2638-2650.

13. Hemmings HC Jr, Akabas MH, Goldstein PA, Trudell JR, Orser BA, Harrison NL. Emerging molecular mechanisms of general anesthetic action. Trends Pharmacol Sci. 2005;26(10):503-510.

14. Antognini JF, Schwartz K. Exaggerated anesthetic requirements in the preferentially anesthetized brain. Anesthesiology. 1993;79(6):1244-1249.

15. Brown EN, Purdon PL, Van Dort CJ. General anesthesia and altered states of arousal: a systems neuroscience analysis. Annu Rev Neurosci. 2011;34:601-628.

16. Schwartzkroin PA. Basic mechanisms of epileptogenesis. In: Wyllie E, ed. The Treatment of Epilepsy. Philadelphia, Pennsylvania, USA: Lea and Febiger; 1993:83-98.

17. Mirsattari SM, Sharpe MD, Young GB. Treatment of refractory status epilepticus with inhalational anesthetic agents isoflurane and desflurane. Arch Neurol. 2004;61(8):1254-1259.
18. Bai D, Pennefather PS, MacDonald JF, Orser BA. The general anesthetic propofol slows deactivation and desensitization of GABA(A) receptors. J Neurosci. 1999;19(24):10635-10646.

19. Ching S, Purdon PL, Vijayan S, Kopell NJ, Brown EN. A neurophysiological-metabolic model for burst suppression. Proc Natl Acad Sci U S A. 2012; 109(8):3095-3100.

20. Furtmüller R, et al. Tranexamic acid, a widely used antifibrinolytic agent, causes convulsions by a gamma-aminobutyric acid(A) receptor antagonistic effect. J Pharmacol Exp Ther. 2002;301(1):168-173.

21. Neville MJ, Butterworth J, James RL, Hammon JW, Stump DA. Similar neurobehavioral outcome after valve or coronary artery operations despite differing carotid emoblic counts. J Thorac Cardiovasc Surg. 2001;121(1):125-136.

22. Makarovsky I, et al. Strychnine-a killer from the past. Isr Med Assoc J. 2008;10(2):142-145.

\title{
Are epsins a therapeutic target for tumor angiogenesis? \\ Nancy Klauber-DeMore
}

Department of Surgery, University of North Carolina at Chapel Hill, Chapel Hill, North Carolina, USA.

\begin{abstract}
Solid tumor growth requires the formation of new blood vessels to supply nutrients and oxygen to the malignant cells; one approach to cancer therapy is to block this process by inhibiting VEGF signaling. In this issue of the JCI, Pasula et al. demonstrate a surprising role of epsins - proteins involved in endocytosis - in tumor angiogenesis via their modulation of VEGF signaling. Their findings suggest that these proteins might represent a new target for the development of cancer therapeutics.
\end{abstract}

Angiogenesis is the formation of new capillary blood vessels and is a critical component of solid tumor growth (1). Once a new tumor reaches just a few cubic millimeters in size, further growth must be preceded by angiogenesis. Tumor cells secrete soluble factors that stimulate vessel growth and/or suppress factors that prevent angiogenesis. These factors act upon endothelial cells to promote their proliferation and migration, resulting in sprouting and tube formation; those tubes then develop into vessels.

Although tumor angiogenesis can be understood as a process required to sustain a cancer's blood supply, the vascular network induced as a result of tumor angiogenesis is highly aberrant, altering the tumor microenvironment and profoundly influencing the manner in which cancers grow, escape the host's immune system,

Conflict of interest: The author has declared that no conflict of interest exists.

Citation for this article: J Clin Invest. 2012; 122(12):4341-4343. doi:10.1172/JCI66171. and metastasize (2). Unlike the organized microvasculature of normal tissue, tumor microvessels are dilated and tortuous, with disorganized patterns of interconnection and branching (3). The erratic tumor vasculature and the resultant hypoxia have additional consequences for tumors: cancer cells undergo epigenetic changes in hypoxic conditions that accelerate their malignant phenotype and the epithelial-to-mesenchymal transition, producing a greater metastatic potential (2). In addition, the cytotoxic functions of immune cells that infiltrate a tumor are compromised in hypoxic and low $\mathrm{pH}$ conditions, further contributing to the malignant phenotype (4).

\section{VEGF family}

An essential mediator of angiogenesis is the VEGF family, which consists of five family members of secreted proteins (VEGFA, VEGFB, VEGFC, VEGFD, VEGFE, and PDGF) (5), that bind and activate three receptor tyrosine kinases (VEGFR1, -2, and -3) (6) which in turn mediate cell sig- naling. VEGFR2 is the key mediator of VEGF-stimulated tumor angiogenesis. When VEGF ligands VEGFR2, the receptor is phosphorylated and activates downstream signaling molecules, resulting in endothelial cell proliferation, migration, tube formation, and the induction of antiapoptotic gene expression (7). VEGF signaling also causes tortuous vasculature formation and vascular leakage in tumors.

\section{Inhibiting VEGF is a therapeutic strategy to inhibit tumor growth}

With the discovery of VEGF as a major driver of tumor angiogenesis, efforts have focused on the development of therapeutics to inhibit VEGF activity, with the goal of inducing tumor regression by starvation. In 2004, a humanized monoclonal antibody to VEGFA, bevacizumab (Avastin; Genentech), became the first FDAapproved antiangiogenic drug in the United States (8). It was approved as a first-line treatment agent for metastatic colorectal cancer, in combination with 5-fluorouracil (9), and was subsequently approved for treatment of metastatic non-squamouscell lung cancer, breast cancer, and glioblastoma multiforme (10). FDA approval was withdrawn for metastatic breast cancer because follow-up studies failed to show an improvement in overall survival. Additional FDA-approved drugs that block VEGF 